\title{
Selective estrogen receptor modulator BC-1 activates antioxidant signaling pathway in vitro via formation of reactive metabolites
}

\author{
Bo-lan $\mathrm{YU}^{1, *}$, Zi-xin MAI ${ }^{1}$, Xu-xiang LIU², Zhao-feng HUANG ${ }^{2, *}$
}

\author{
${ }^{1}$ Key Laboratory of Reproduction and Genetics of Guangdong Higher Education Institute, Third Affiliated Hospital of Guangzhou \\ Medical University, Guangzhou 510150, China; ${ }^{2}$ Institute of Human Virology, Zhongshan School of Medicine, Sun Yat-sen University, \\ Guangzhou 510080, China
}

Aim: Benzothiophene compounds are selective estrogen receptor modulators (SERMs), which are recently found to activate antioxidant signaling. In this study the molecular mechanisms of antioxidant signaling activation by benzothiophene compound BC-1 were investigated.

Methods: HepG2 cells were stably transfected with antioxidant response element (ARE)-luciferase reporter (HepG2-ARE cells). The expression of nuclear factor erythroid 2-related factor 2 (Nrf2) in HepG2-ARE cells was suppressed using siRNA. The metabolites of BC-1 in rat liver microsome incubation were analyzed using LC-UV and LC-MS.

Results: Addition of BC-1 ( $5 \mu \mathrm{mol} / \mathrm{L})$ in HepG2-ARE cells resulted in a 17-fold increase of ARE-luciferase activity. Pretreatment with the estrogen receptor agonist $\mathrm{E}_{2}(5 \mu \mathrm{mol} / \mathrm{L})$ or antagonist ICI 182,780 (5 $\left.\mu \mathrm{mol} / \mathrm{L}\right)$ did not affect BC-1-induced ARE-luciferase activity. However, transfection of the cells with anti-Nrf2 siRNA suppressed this effect by $79 \%$. Addition of BC-1 in rat microsome incubation resulted in formation of di-quinone methides and o-quinones, followed by formation of GSH conjugates. BC-1 analogues with hydrogen (BC-2) or fluorine (BC-3) at the $4^{\prime}$ position did not form the di-quinone methides. Both $\mathrm{BC}-2$ and $\mathrm{BC}-3$ showed comparable estrogenic activity with BC-1, but did not induce ARE-luciferase activity in HepG2-ARE cells.

Conclusion: Benzothiophene compound BC-1 activates ARE signaling via reactive metabolite formation that is independent of estrogen receptors.

Keywords: selective estrogen receptor modulator; benzothiophene compound; $\mathrm{E}_{2}$; ICI 182,780; antioxidant response element (ARE); reactive metabolites; quinoid formation; nuclear factor erythroid 2-related factor 2 (Nrf2); siRNA

Acta Pharmacologica Sinica (2013) 34: 373-379; doi: 10.1038/aps.2012.168; published online 21 Jan 2013

\section{Introduction}

Selective estrogen receptor modulators (SERMs) are clinically important for the treatment and prevention of breast cancer as well as for therapy of postmenopausal osteoporosis ${ }^{[1]}$. The first generation triphenylethylene SERM, tamoxifen, significantly decreases the incidence of breast cancer in high-risk women and has good efficacy as a first line of defense in breast cancer therapy ${ }^{[2]}$. However, its associated side effects such as an increased incidence of endometrial cancer limit the longterm usage of this drug ${ }^{[1,2]}$.

Benzothiophene SERMs, including raloxifene and arzoxifene

\footnotetext{
* To whom correspondence should be addressed.

E-mail yubolan@gmail.com (Bo-lan YU); hzhaof@mail.sysu.edu.cn (Zhao-feng HUANG)

Received 2012-08-06 Accepted 2012-11-12
}

(Figure 1), have demonstrated a more favorable therapeutic and safety profile in clinical as well as preclinical studies ${ }^{[1]}$. Raloxifene is a second generation SERM that has been widely used for the prevention and treatment of postmenopausal osteoporosis and for breast cancer chemoprevention in highrisk women ${ }^{[3,4]}$. Arzoxifene comes from a new generation of benzothiophene SERMs ${ }^{[5]}$, and both arzoxifene and its active metabolite desmethylarzoxifene (DMA) have a higher antiestrogenic potency than tamoxifen and better bioavailability than raloxifene ${ }^{[6]}$. Due to the absence of agonist activity in the endometrium, the presence of antiestrogenicity in the breast and estrogenicity in bones ${ }^{[1]}$, it is believed that benzothiophene SERMs are safer for long-term treatment compared to tamoxifen. Currently, there are no reports suggesting that raloxifene and arzoxifene could cause DNA damage and carcinogenesis. In contrast, it has been reported that reactive quinoid metabo- 
A

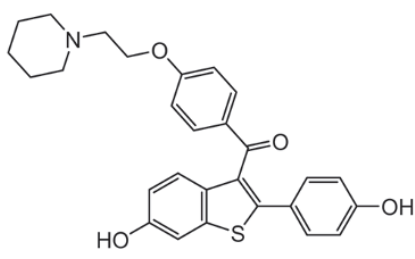

Raloxifene

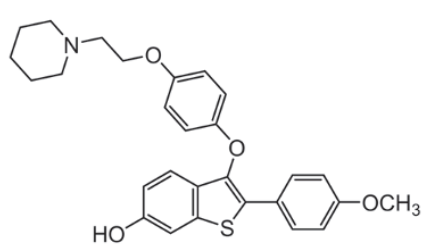

Arzoxifene

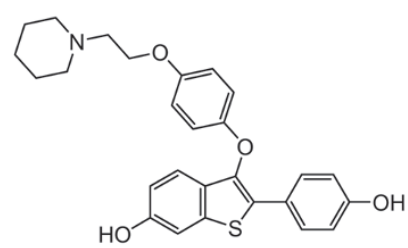

Desmethylarzoxifene

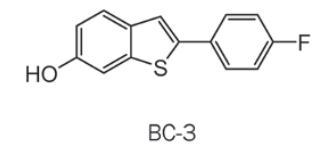

BC-3
B

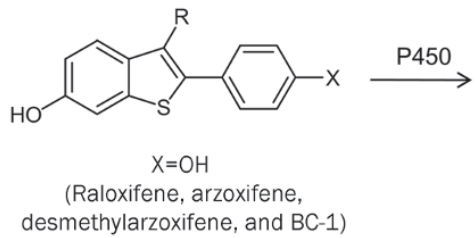

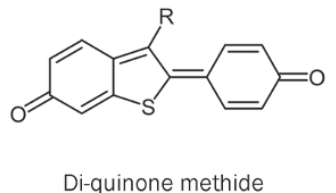

Di-quinone methide

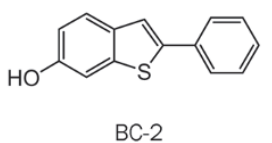

Figure 1. Structures of benzothiophene compounds. (A) Chemical structures of the benzothiophene SERMs raloxifene, arzoxifene, and desmethylarzoxifene, the benzothiophene compound BC-1 and its analogues BC-2 and BC-3. (B) Formation of di-quinone methide from benzothiophene compounds.

lites formed from benzothiophene SERMs are capable of inducing cytoprotective quinone reductase (NQO1) activity in both cell and animal models ${ }^{[7]}$.

Induction of cytoprotective enzymes is largely mediated by the antioxidant responsive element (ARE), which promotes the transcription of many antioxidant genes such as NQO1, superoxide dismutase isoenzymes (SOD) and glutathione S-transferase $(\mathrm{GST})^{[8,9]}$. The cellular sensor protein Kelch-like ECH-associated protein 1 (Keap1) contains large numbers of sulfhydryl groups that can be modified by oxidants and electrophiles ${ }^{[10,11]}$. Under basal conditions, Keap1 associates with the key transcriptional factor nuclear factor erythroid 2-related factor 2 (Nrf2) and targets it for degradation ${ }^{[12]}$. When Keap1 is modified by either oxidative or electrophilic reagents, proteolysis is inhibited, and the dissociated Nrf2 translocates to the nucleus, binds to ARE, and promotes phase II enzyme gene expression ${ }^{[12]}$. Therefore, oxidants and electrophiles might initiate the inducer signal by modifying Keap1 sulfhydryl groups, which can be further transduced by the Keap1-Nrf2 signaling pathway to activate cellular ARE.

All SERMs and their active metabolites share common polyaromatic scaffolds that are able to form quinoids ${ }^{[13]}$. These quinoid metabolites are reactive electrophiles that can covalently modify cellular macromolecules and either cause genotoxicity and cytotoxicity or contribute to cytoprotection ${ }^{[14]}$. It has been reported that in the rat liver and intestine, tamoxifen could induce the phase II enzymes aryl sulfotransferase and hydroxysteroid sulfotransferase ${ }^{[15]}$. Separate studies reported that 4-hydroxytamoxifen and raloxifene could induce NQO1 in MCF-7 cells via an estrogen receptor (ER) dependent mechanism ${ }^{[16,17]}$. In our previous work, it was found that arzoxifene and its active metabolite DMA could induce NQO1 independently of ER pathways in both cells and rat models ${ }^{[7]}$. These data suggest that SERMs might produce beneficial quinoid electrophiles and activate the Keap1-Nrf2-ARE pathways. Further studies showed that a benzothiophene core compound could significantly induce NQO1 activity and ARE signaling compared to arzoxifene and $\mathrm{DMA}^{[7]}$, which might serve as a potential benzothiophene SERM for chemoprevention. However, the detailed mechanism of its antioxidant signaling induction is still unclear.

In this study, the potential mechanism of ARE induction by the compound BC-1 was investigated based on the hypothesis that its reactive metabolites could modify Keap1 and thus activate ARE. Using cell models, the induction capability of the ARE signaling pathway by BC-1 was measured, the possible regulatory factors (including ER and Nrf2) were examined, and the in vitro quinoid formation of BC-1 was analyzed. Compared with two analogues of BC-1 that can not form reactive di-quinone methides (Figure 1), it was assumed that this quinoid formation of BC-1 played a key role in ARE induction via Keap1-Nrf2 and, therefore, suggested a novel mechanism of antioxidant signaling activation by benzothiophene SERMs via reactive metabolite formation.

\section{Materials and methods}

\section{Reagents}

All chemicals and reagents were purchased from SigmaAldrich (St Louis, MO, USA) unless stated otherwise. Cell culture media and supplements were obtained from Life Technol- 
ogies (Grand Island, NY, USA). The luciferase substrate was obtained from Promega (Madison, WI, USA). The BCA assay kit was obtained from Thermo Fisher Scientific (Rockford, IL, USA). The benzothiophene compounds BC-1, BC-2, BC-3 were synthesized by J\&K Scientific Ltd (Beijing, China) as described in the literature ${ }^{[7]}$.

\section{Cell culture}

Hepa1c1c7 murine hepatoma cells were cultured in a-MEM with $1 \%$ penicillin-streptomycin and $10 \%$ fetal bovine serum (FBS). HepG2 cells stably transfected with ARE-luciferase reporter were cultured in F-12 medium with 10\% FBS, 1\% penicillin-streptomycin, $1 \%$ essential amino acids, and $0.2 \mathrm{mg} / \mathrm{mL}$ insulin. All cells were incubated in $5 \% \mathrm{CO}_{2}$ at $37^{\circ} \mathrm{C}$, and the medium was replaced every 2-3 d.

\section{ARE-luciferase reporter assay}

HepG2 cells stably transfected with ARE-luciferase reporter were plated in a 24 -well plate $\left(2 \times 10^{4}\right.$ cells per well), and cells were treated with either control (DMSO) or test compounds and incubated for various lengths of time. The luciferase activity was determined according to the protocol provided by the manufacturer (Promega). Briefly, cells were washed with cold phosphate buffered saline (PBS) twice and lysed with 500 $\mu \mathrm{L}$ of reporter lysis buffer. After centrifugation, $20 \mu \mathrm{L}$ of the supernatant was used for measurement of the luciferase activity and normalized to its protein concentration using the BCA assay. The data were obtained from three separate experiments and expressed as fold-induction over the control.

\section{siRNA interference}

Small interfering RNA (siRNA) experiments were performed on HepG2-ARE cells using anti-Nrf2 and nonspecific scramble siRNA lentiviral transfection particles (Sigma). NM_006164.21144s1c1 (5'-CCGGCCGGCATTTCACTAAACACAACTCGAGTTGTGTTTAGTGAAATGCCGGTTTTT-3') was selected based on preliminary data. HepG2 cells stably expressing ARE luciferase report were plated in 96 -well plates $\left(0.5 \times 10^{4}\right.$ cells per well) one day prior to transfection. On d 2 when the cell confluency reached $70 \%-80 \%$, the cell culture medium was changed, and $150 \mu \mathrm{L}$ fresh medium containing hexadimethrine bromide $\left(4 \mathrm{mg} / \mathrm{mL}\right.$ stock solution in $\mathrm{ddH}_{2} \mathrm{O}$, final concentration $8 \mu \mathrm{g} / \mathrm{mL}$ ) was added to the cells and gently swirled in the plate to mix. Lentiviral particles were thawed at room temperature and added to cells, and plates were gently swirled to mix. Plates were centrifuged at $500 \times \mathrm{g}$ for $1 \mathrm{~h}$ at room temperature and incubated at $37^{\circ} \mathrm{C}$ for $16 \mathrm{~h}$. The cell culture medium was changed on $\mathrm{d} 3$, and on $\mathrm{d} 4$, the compounds (5 $\mu \mathrm{mol} / \mathrm{L})$ were added to the cells, and the luciferase assay was performed after a $6 \mathrm{~h}$ incubation.

\section{Rat liver microsomal incubation}

A solution containing the test compounds, rat liver microsomes $(1.0 \mathrm{mg} / \mathrm{mL})$, reduced $L$-glutathione (GSH, 500 $\mu \mathrm{mol} / \mathrm{L})$ and reduced $\beta$-Nicotinamide adenine dinucleotide 2'-phosphate (NADPH, $1 \mathrm{mmol} / \mathrm{L}$ ) in $50 \mathrm{mmol} / \mathrm{L}$ phosphate buffer (pH 7.4, $500 \mu \mathrm{L}$ total volume) was incubated for $30 \mathrm{~min}$ at $37^{\circ} \mathrm{C}$. For comparisons, either GSH or NADPH were omitted as controls. The reactions were terminated by chilling in an ice bath followed by the addition of methanol $(500 \mu \mathrm{L})$ containing perchloric acid $(50 \mu \mathrm{L} / \mathrm{mL})$. The reaction mixtures were centrifuged at $15000 \times g$ for $10 \mathrm{~min}$, and aliquots $(80 \mu \mathrm{L})$ of the supernatant were analyzed using liquid chromatography tandem mass spectrometry (LC-MS/MS).

\section{In vitro metabolites identification}

Analytical liquid chromatography-ultraviolet (LC-UV) analysis was performed using a $5 \mu \mathrm{mol} / \mathrm{L}, 4.6 \mathrm{~mm} \times 250 \mathrm{~mm}$ Aglient Eclipse XDB C18 column at a flow rate of $1.0 \mathrm{~mL} / \mathrm{min}$. The mobile phase $\mathrm{A}$ consisted of $\mathrm{H}_{2} \mathrm{O}$ with $0.1 \%$ formic acid, and the mobile phase $\mathrm{B}$ was acetonitrile with $0.1 \%$ formic acid. The initial mobile phase consisted of $10 \%$ B followed by a 40 min linear gradient to $30 \% \mathrm{~B}$, then a linear gradient from $30 \%$ to $60 \%$ B for $10 \mathrm{~min}$, and finally a $5 \mathrm{~min}$ gradient from $60 \%$ to $90 \%$ B. The LC-MS/MS spectra were obtained using a Shimadzu LCMS-IT-TOF mass spectrometer (Kyoto, Japan).

\section{Statistical analysis}

Student's t-test and one-way ANOVA analysis with Tukey's multiple comparison test were performed using GraphPad Prism version 4.00 for Windows (La Jolla, CA, USA).

\section{Results}

Estrogen receptor independent mechanism for ARE activation in cells

The potential of BC-1 to activate ARE was comparable to 4-bromoflavone, which is an effective chemopreventive reagent, as observed in our previous study ${ }^{[7]}$. The data from our pilot study also showed that a dose of BC-1 between 1 $\mu \mathrm{mol} / \mathrm{L}$ and $20 \mu \mathrm{mol} / \mathrm{L}$ could activate ARE (unpublished data). Because $5 \mu \mathrm{mol} / \mathrm{L}$ of BC- 1 could strongly activate ARE but was below its $\mathrm{IC}_{50}(26 \mu \mathrm{mol} / \mathrm{L})$, this concentration was applied to all cellular experiments in this study.

The roles of ERs, especially ER $\beta$, in the SERM-induced phase II enzyme induction are controversial ${ }^{[16,17]}$. To investigate the role of ERs in BC-1 induced ARE activation, HepG2-ARE cells were pretreated with either the ER agonist $E_{2}$ or antagonist ICI 182,780 prior to the addition of BC-1. ARE induction was examined in cells that were treated with vehicle control, $\mathrm{E}_{2}$, ICI 182,780, and BC-1 individually, as well as cells that were treated with various combinations of these compounds. The results indicated that $\mathrm{E}_{2}$, ICI 182,780, and vehicle DMSO could not induce ARE activation, and BC-1 was the only compound that could induce activity alone (Figure 2). BC-1 was a potent inducer of ARE-luciferase activity, with a $5 \mu \mathrm{mol} / \mathrm{L}$ dose resulting in a 17-fold increase compared to the control. Cotreatment of BC-1 with either $\mathrm{E}_{2}$ or ICI 182,780 neither inhibited nor enhanced BC-1 induced ARE activation significantly (Figure 2). Therefore, it is suggested that ERs are not involved in BC-1 induced ARE activation in HepG2 cells. 


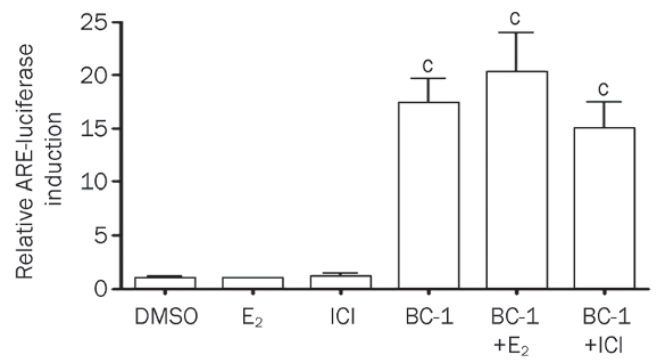

Figure 2. The effects of an ER agonist and antagonist on BC-1 induced ARE luciferase activity. HepG2-ARE cells were treated with vehicle, the ER agonist $E_{2}(5 \mu \mathrm{mol} / \mathrm{L})$, the ER antagonist ICl 182,780 (5 $\left.\mu \mathrm{mol} / \mathrm{L}\right), \mathrm{BC}-1$ $(5 \mu \mathrm{mol} / \mathrm{L})$, and various combinations of these compounds. Luciferase activity was measured after treatment for $24 \mathrm{~h}$. The results were expressed as the mean \pm SD and compared using one-way ANOVA with Tukey's post-test. ${ }^{c} P<0.01$ compared to the controls.

\section{Involvement of Nrf2 in the ARE induction by BC-1}

HepG2-ARE cells were further transduced with siRNA lentiviral particles targeting Nrf2 to examine the role of Nrf2 in ARE activation by BC-1. The results showed that the transfection of anti-Nrf2 siRNA significantly decreased BC-1 induced ARE luciferase activity compared to the cells transduced with control siRNA, whereas no difference was observed in the cells receiving DMSO treatment (Figure 3).

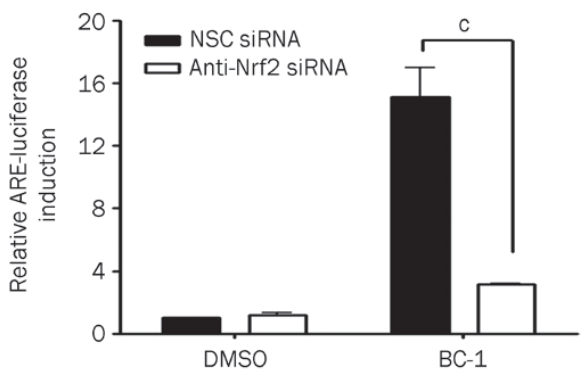

Figure 3. Effects of anti-Nrf2 siRNA on BC-1 induced ARE luciferase activity. HepG2-ARE cells were treated with either DMSO or $5 \mu \mathrm{mol} / \mathrm{L}$ BC-1 and transfeced with either anti-Nrf2 or non-specific scrambled siRNA (NSC siRNA) lentiviral particles. Luciferase activity was measured at $24 \mathrm{~h}$ post transfection, and comparisons were made between the controls and the treated cells. ${ }^{c} P<0.01$ compared between the different treatments.

Other factors such as mitogen-activated protein kinase (MAPK) and protein kinase C (PKC) are known to be involved in ARE activation ${ }^{[18]}$. Using specific inhibitors to MAPK and PKC (U0126 and staurosporine, respectively), it was observed that inhibition of either kinase had no effect on BC-1 induced ARE activity (unpublished data). Therefore, whether these kinases play a role in BC-1 induced ARE deserves future investigation.

Quinoid formation of $\mathrm{BC}-1$ in rat liver microsomes

The benzothiophene core compound BC-1 contains a pol- yaromatic phenolic scaffold, which can be bioactivated into quinoids. To investigate whether $\mathrm{BC}-1$ can generate quinoid metabolites, rat liver microsomes either with or without GSH were incubated with $10 \mu \mathrm{mol} / \mathrm{L}$ of BC-1. Unstable reactive quinoid intermediates formed in incubations were trapped with GSH, and then extracted and analyzed by LC-UV and LC-MS/MS (Figure 4 and Supplemental Figures 1-3).

In the LC-UV chromatogram, there were two major types of quinoids: di-quinone methide and o-quinone, both of which were identified as reactive metabolites of BC-1 in microsomal incubations (Figure 4A). These quinoids went on to form diquinone methide GSH conjugates, o-quinone GSH conjugates, and $o$-quinone di-GSH conjugates, all of which were identified by LC-MS/MS (Supplemental Figures 1-3).

A

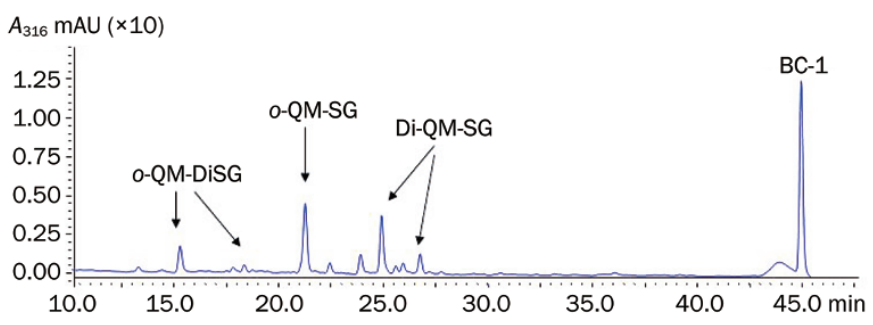

B

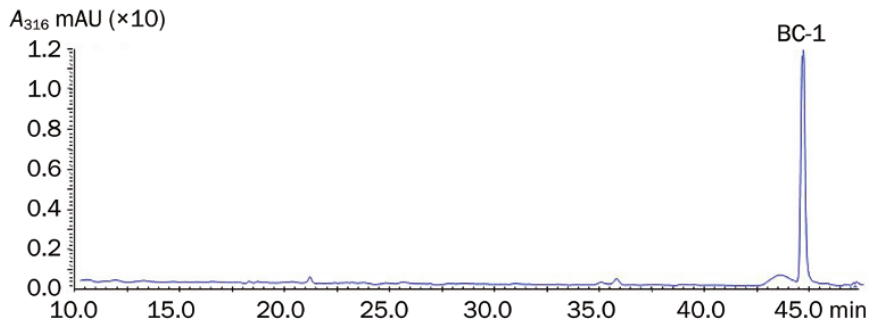

Figure 4. Quinone formation of $\mathrm{BC}-1$ in rat liver microsomes. LC-UV chromatogram of BC-1 $(10 \mu \mathrm{mol} / \mathrm{L})$ incubated with rat liver microsomes in the presence $(A)$ and absence $(B)$ of GSH.

The major GSH conjugates, BC-1 di-quinone methide monoGSH conjugates, were identified based on the detection of $[\mathrm{M}+\mathrm{H}]^{+}$at $\mathrm{m} / z 548$ at 24.5 min (Supplemental Figure 1). Subsequent collision-induced dissociation (CID) of this ion produced the most abundant ions at $m / z 419$, which corresponds to the neutral loss of anhydroglutamic acid (129 Da) from GSH. Other fragment ions included those at $m / z 273$ and $m / z 473$, which were derived from the cleavage of $\mathrm{S}-\mathrm{CH}_{2}$ bond within the cysteine residue (275 Da) and the neutral loss of glycine $(75 \mathrm{Da})$ from parent compound, respectively. These fragmentations have been considered to be the unique CID fragmentations of GSH for confirmation of the formation of drug-GSH conjugates ${ }^{[19,20]}$.

BC-1 also formed o-quinone mono GSH conjugates based on the detection of its protonated ions at $\mathrm{m} / z 564$ at $21.0 \mathrm{~min}$. CID of this ion also gave similar fragmentation derived from GSH 
as the di-quinone methide GSH conjugates (Supplemental Figure 2). The base peak at $m / z 435$ resulted from the neutral loss of anhydroglutamic acid, the ion at $\mathrm{m} / \mathrm{z} 418$ from the neutral loss of glutamine (146 Da), and the ion at $\mathrm{m} / \mathrm{z} 343$ from the loss of their combination. The ion at $m / z 289$ was derived from the cleavage of $\mathrm{S}-\mathrm{CH}_{2}$ bond within the cysteine residue.

BC-1 o-quinone di-GSH conjugates were identified based on the detection of $[\mathrm{M}+2 \mathrm{H}]^{2+}$ at $m / z 435$ at $15.0 \mathrm{~min}$ (Supplemental Figure 3). CID fragmentation of this ion also gave similar fragment ions derived from the loss of different moieties of GSH.

Thus, BC-1 could largely form reactive quinoids in microsomal incubations, which were trapped by GSH. The formation of similar reactive intermediates from BC-1 in cells and in vivo is possible, which might contribute to ARE activation via reaction with cellular macromolecules. However, the exact structures of the formed conjugates are unclear because BC-1-derived quinoids could form several GSH conjugates, including isomers. Detailed investigations on BC-1's GSH conjugates may be warranted in the future.

\section{Modulation of ARE induction activity by BC-1 analogues}

To modulate the antioxidant activity of BC-1, a series of analogues were synthesized, in which the hydroxyl group at the $4^{\prime}$ position of BC-1 was replaced with either hydrogen (BC-2) or fluorine (BC-3) (Figure 1). Both of the analogues had comparable estrogenic activity with BC-1 in cells (Supplemental Table 1); however, BC-2 and BC-3 could not form di-quinone methides (Figure 1).

Induction of ARE activation in HepG2 cells by BC-1 and its analogues at $5 \mu \mathrm{mol} / \mathrm{L}$ were measured (Figure 5). As a potent phase II enzyme inducer, BC-1 activated ARE approximately 15-fold compared to the control; however, both BC-2 and BC-3 showed no significant activation of ARE. Therefore, these data suggested a correlation between ARE activation by this benzothiophene compound and its ability to form reactive diquinone methides.

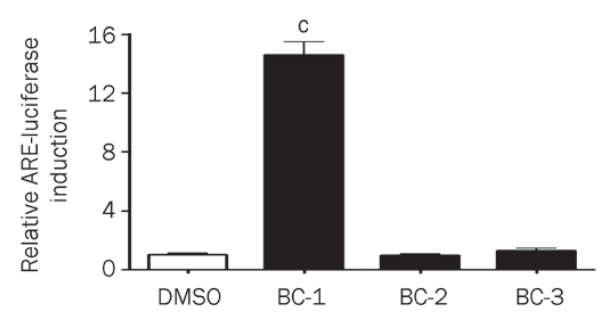

Figure 5. Induction of ARE signaling pathways by BC-1 analogues. HepG2-ARE cells were incubation with either BC-1 (5 $\mu \mathrm{mol} / \mathrm{L})$ or one of its analogues (BC-2 and BC-3, $5 \mu \mathrm{mol} / \mathrm{L})$. Luciferase activity was measured after treatment for $24 \mathrm{~h}$ and compared to vehicle control (DMSO). ${ }^{\mathrm{c}} \mathrm{P}<0.01$ compared to controls.

\section{Discussion}

The predicted long-term clinical use of SERMs and their risk of carcinogenesis require a complete understanding of their detrimental and beneficial effects in humans. Benzothiophene SERMs and their active metabolites contain polyaromatic phenol structures that can form quinoids ${ }^{[14,21-24]}$, which can covalently modify cellular macromolecules and cause different biological consequences ${ }^{[13]}$. In this study, we found that reactive metabolites were formed from compound BC-1, which is the core structure of benzothiophene SERMs (Figure 1), and activated ARE signaling pathways via reactive electrophilic metabolites (Figures 1-5). These data strongly support the concept that appropriate benzothiophene compounds can act as chemopreventive agents via the Keap1-Nrf2-ARE pathway and allow for the correlation of benzothiophene compound structure with the potential to induce chemopreventive mechanisms.

A previous study reported that the benzothiophene core compound BC-1 could greatly activate ARE signaling ${ }^{[7]}$, but a detailed study on the mechanism of its activation had not been performed. Our data supported the theory that the induction of ARE by BC-1 was ER independent (Figure 2) despite BC-1 being an effective ER modulator (Supplemental Table $1)$. Interestingly, the chemopreventive effects of the benzothiophene SERM arzoxifene toward ER negative breast cancer have been reported by the Sporn group ${ }^{[25]}$. Their data demonstrated that a single treatment of arzoxifene significantly delayed the development of ER negative tumors in a transgenic mouse model ${ }^{[25]}$, which suggests that the chemopreventive effect of arzoxifene in this breast cancer model was independent of the ER signaling pathway. Though it is uncertain if the induction of antioxidant signaling by arzoxifene contributes to its anticarcinogenic effects, these data suggested that the beneficial biological effects of benzothiophene compounds may involve mechanisms other than ER pathways.

This study showed that the activation of Keap1 was demonstrated to be important for the ARE induction by BC-1 (Figure $3)$. ARE activation is one of the most important chemopreventive mechanisms; many inducible antioxidant enzymes such as NQO1, GST, and SOD contain an ARE structure and provide cytoprotection by the detoxification of small molecules ${ }^{[8,9]}$. Many chemopreventive agents such as sulforaphane and Michael acceptors have been shown to chemically modify the cysteine residues of the redox sensor protein Keap1 and lead to the activation of ARE by Nrf2 ${ }^{[18,26]}$. Animal studies using Keap1 and Nrf2 knockouts indicated the importance of the Keap1-Nrf2-ARE pathway for antioxidant enzyme induction by sulforaphane and other agents ${ }^{[27]}$. In this study, Nrf2 was also shown to be involved in ARE activation by BC-1 (Figure $3)$, suggesting that the sensor protein Keap1, which regulates Nrf2 translocation, is modified. Further research is required to differentiate the exact mechanisms invoked by BC-1, including the roles of the MAPK and PKC pathways.

The benzothiophene compound BC-1 could form large amounts of di-quinone methide metabolites that are trapped by GSH when incubated with rat liver microsomes, as shown in this study (Figure 4, Supplemental Figures 1-3). These quinoids are reactive electrophiles and are potential oxidation/ alkylation agents for biomolecules such as proteins, DNA, 
and GSH. There is controversy over the degree of specificity of covalent modification by electrophiles, but the study by the Liebler group suggested that the protein modifications were not random and certain protein families were particularly susceptible to alkylation ${ }^{[28]}$. It has been reported that quinoids formed from benzothiophene SERMs (including raloxifene and arzoxifene) participate in enzyme inhibition and protein modification ${ }^{[23,29-32]}$. For instance, irreversible inhibition of the cytochrome P450 enzyme CYP3A4 and alkylation of proteins in rat liver microsomes by raloxifene were observed ${ }^{[23,29]}$. Recent studies reported a modification on either Cys239 or Tyr75 of CYP3A4 by raloxifene ${ }^{[30-32]}$, which may play critical roles in the time-dependent inactivation of CYP3A4 by raloxifene. Therefore, after its bioactivation to form di-quinone methides, BC-1 might be responsible for the reactivity towards the Keap1 sensor required to induce ARE.

We elucidated that the formation of reactive di-quinone methides of benzothiophene compounds might play the central role in ARE activation. Benzothiophene SERMs were able to form several types of quinoids, and di-quinone methides were the most abundant electrophilic metabolites formed from benzothiophene compounds ${ }^{[13]}$. In vitro, the half-life of the DMA di-quinone methide is approximately $15 \mathrm{~s}^{[22,33]}$, and the half-life of BC-1 di-quinone methide is approximately 20 $\mathrm{s}$ (unpublished data). According to Thompson et al, quinoids with half-lives in the range of $10 \mathrm{~s}$ to $10 \mathrm{~min}$ have time to diffuse away from the site of formation to react with cellular nucleophiles ${ }^{[34]}$. Di-quinone methides prefer to react with either sulfur nucleophiles such as GSH or protein cysteine sulfhydryl groups, and may serve as the chemical trigger for ARE activation. The importance of di-quinone methide formation from BC-1 for its ARE induction was further proven by comparison with its analogues (Figures 1 and 5). When the 4'-OH group of BC-1 was replaced by hydrogen and fluoride (BC-2 and BC-3, respectively), oxidation of the compounds to form a di-quinoid methide was blocked ${ }^{[21]}$, and ARE induction in the cells was completely lost in both BC-2 and BC-3 treatments (Figure 5). Therefore, the formation of reactive di-quinoid methides from BC-1 might be responsible for ARE induction, and preventing their formation would result in the loss of its putative beneficial effects via induction of antioxidant signaling.

Though BC-1 represented the major aromatic structure of raloxifene and arzoxifene, it was a much stronger inducer of ARE signaling compared to both analogues ${ }^{[7]}$. A potential explanation for the weak induction by arzoxifene and raloxifene is that direct interaction of the quinoid with the sensor protein is required. The steric hindrance due to the SERM side arm in both raloxifene and arzoxifene would interfere with the interaction of drug metabolites and the sensor proteins (Figure 1). It is also known that raloxifene could form catechols, $o$-quinones and di-quinone methods, but the half-life of the raloxifene di-quinone methide is only $1 s^{[33]}$. Therefore, the short half-life of raloxifene di-quinone methide may be another explanation for its weak ARE induction. Because the characteristics of quinoid metabolites formed from SERMs are important, engineering a SERM structure to modulate redox activity might influence its biological activity with potential therapeutic significance.

In conclusion, this study showed that the core compound of benzothiophene SERMs, BC-1, could activate ARE signaling pathways via reactive electrophilic metabolite formation. There was no evidence supporting the involvement of the ERs in this process, but the key regulatory factor Nrf2 was demonstrated to be involved in ARE activation by BC-1. These observations suggested a possible correlation between the induction of antioxidant signaling pathways by benzothiophene compounds and their ability to form reactive quinoids; therefore, we have elucidated a novel mechanism of antioxidant signaling activation by benzothiophene compounds via reactive metabolite formation.

\section{Acknowledgements}

This study was supported by the National Natural Science Foundation of China (81102474), the Guangdong Natural Science Foundation (S2011040003768), the Foundation of Guangzhou City Science and Technology Administration (2012J4300089), and the Foundation from Guangzhou Medical University (2011C09).

\section{Author contribution}

Bo-lan YU conceived the study and wrote the manuscript; Zi-xin MAI conducted the cell experiments and analyzed the data; Xu-xiang LIU performed the LC-MS/MS experiments and analyzed the data; Zhao-feng HUANG supervised the entire study and revised the manuscript.

\section{Supplementary information}

Supplementary information is available at website of Acta Pharmacologica Sinica on NPG.

\section{References}

1 Shelly W, Draper MW, Krishnan V, Wong M, Jaffe RB. Selective estrogen receptor modulators: an update on recent clinical findings. Obstet Gynecol Surv 2008; 63: 163-81.

2 Jordan VC. Tamoxifen $(\mathrm{ICl} 46,474)$ as a targeted therapy to treat and prevent breast cancer. Br J Pharmacol 2006; 147: S269-76.

3 Bolognese M, Krege JH, Utian WH, Feldman R, Broy S, Meats DL, et al. Effects of arzoxifene on bone mineral density and endometrium in postmenopausal women with normal or low bone mass. J Clin Endocrinol Metab 2009; 94: 2284-9.

4 Vogel VG, Costantino JP, Wickerham DL, Cronin WM, Cecchini RS, Atkins JN, et al. Effects of tamoxifen vs raloxifene on the risk of developing invasive breast cancer and other disease outcomes: the NSABP Study of Tamoxifen and Raloxifene (STAR) P-2 trial. JAMA 2006; 295: 2727-41.

5 Sporn MB. Arzoxifene: a promising new selective estrogen receptor modulator for clinical chemoprevention of breast cancer. Clin Cancer Res 2004; 10: 5313-5.

6 Baselga J, Llombart-Cussac A, Bellet M, Guillem-Porta V, Enas N, Krejcy K, et al. Randomized, double-blind, multicenter trial comparing two doses of arzoxifene (LY353381) in hormone-sensitive advanced or metastatic breast cancer patients. Ann Oncol 2003; 14: 1383-90.

7 Yu B, Dietz BM, Dunlap T, Kastrati I, Lantvit DD, Overk CR, et al. 
Structural modulation of reactivity/activity in design of improved benzothiophene selective estrogen receptor modulators: induction of chemopreventive mechanisms. Mol Cancer Ther 2007; 6: 2418-28.

8 Kensler TW, Wakabayashi N, Biswal S. Cell survival responses to environmental stresses via the Keap1-Nrf2-ARE pathway. Annu Rev Pharmacol Toxicol 2007; 47: 89-116.

9 Wakabayashi N, Slocum SL, Skoko JJ, Shin S, Kensler TW. When NRF2 talks, who's listening? Antioxid Redox Signal 2010; 13: 164963.

10 Nguyen T, Sherratt PJ, Huang HC, Yang CS, Pickett CB. Increased protein stability as a mechanism that enhances Nrf2-mediated transcriptional activation of the antioxidant response element. Degradation of Nrf2 by the 26 S proteasome. J Biol Chem 2003; 278 : 4536-41.

11 Zhang DD, Hannink M. Distinct cysteine residues in Keap1 are required for Keap1-dependent ubiquitination of $\mathrm{Nrf2}$ and for stabilization of Nrf2 by chemopreventive agents and oxidative stress. Mol Cell Biol 2003; 23: 8137-51.

12 Holland R, Fishbein JC. Chemistry of the cysteine sensors in Kelchlike ECH-associated protein 1. Antioxid Redox Signal 2010; 13: 1749-61.

13 Dowers TS, Qin ZH, Thatcher GR, Bolton JL. Bioactivation of selective estrogen receptor modulators (SERMs). Chem Res Toxicol 2006; 19: 1125-37.

14 Bolton JL. Quinoids, quinoid radicals, and phenoxyl radicals formed from estrogens and antiestrogens. Toxicology 2002; 177: 55-65.

15 Maiti S, Chen G. Tamoxifen induction of aryl sulfotransferase and hydroxysteroid sulfotransferase in male and female rat liver and intestine. Drug Metab Dispos 2003; 31: 637-44.

16 Bianco NR, Perry G, Smith MA, Templeton DJ, Montano MM. Functional implications of antiestrogen induction of quinone reductase: inhibition of estrogen-induced deoxyribonucleic acid damage. Mol Endocrinol 2003; 17: 1344-55.

17 Montano MM, Chaplin U, Deng H, Mesia-Vela S, Gaikwad N, Zahid M, et al. Protective roles of quinone reductase and tamoxifen against estrogen-induced mammary tumorigenesis. Oncogene 2006; 26: 3587-90.

18 Giudice A, Montella M. Activation of the Nrf2-ARE signaling pathway: a promising strategy in cancer prevention. Bioessays 2006; 28: 16981.

19 Dieckhaus CM, Fernandez-Metzler CL, King R, Krolikowski PH, Baillie TA. Negative ion tandem mass spectrometry for the detection of glutathione conjugates. Chem Res Toxicol 2005; 18: 630-8.

20 Levsen K, Schiebel HM, Behnke B, Dotzer R, Dreher W, Elend M, et al. Structure elucidation of phase II metabolites by tandem mass spectrometry: an overview. J Chromatogr A 2005; 1067: 55-72.

21 Liu H, Bolton JL, Thatcher GR. Chemical modification modulates estrogenic activity, oxidative reactivity, and metabolic stability in $4^{\prime} \mathrm{F}$ DMA, a new benzothiophene selective estrogen receptor modulator. Chem Res Toxicol 2006; 19: 779-87.
22 Liu H, Liu J, van Breemen RB, Thatcher GR, Bolton JL. Bioactivation of the selective estrogen receptor modulator desmethylated arzoxifene to quinoids: $4^{\prime}$-fluoro substitution prevents quinoid formation. Chem Res Toxicol 2005; 18: 162-73.

23 Liu J, Li Q, Yang X, van Breemen RB, Bolton JL, Thatcher GR. Analysis of protein covalent modification by xenobiotics using a covert oxidatively activated tag: raloxifene proof-of-principle study. Chem Res Toxicol 2005; 18: 1485-96.

24 Liu J, Liu H, van Breemen RB, Thatcher GR, Bolton JL. Bioactivation of the selective estrogen receptor modulator acolbifene to quinone methides. Chem Res Toxicol 2005; 18: 174-82.

25 Liby K, Rendi M, Suh N, Royce DB, Risingsong R, Williams CR, et al. The combination of the rexinoid, LG100268, and a selective estrogen receptor modulator, either arzoxifene or acolbifene, synergizes in the prevention and treatment of mammary tumors in an estrogen receptor-negative model of breast cancer. Clin Cancer Res 2006; 12 : 5902-9.

26 Kwak MK, Wakabayashi N, Kensler TW. Chemoprevention through the Keap1-Nrf2 signaling pathway by phase 2 enzyme inducers. Mutat Res 2004; 555: 133-48.

27 Wakabayashi N, Dinkova-Kostova AT, Holtzclaw WD, Kang MI, Kobayashi A, Yamamoto M, et al. Protection against electrophile and oxidant stress by induction of the phase 2 response: fate of cysteines of the Keap1 sensor modified by inducers. Proc Natl Acad Sci U S A 2004; 101: 2040-5.

28 Dennehy MK, Richards KA, Wernke GR, Shyr Y, Liebler DC. Cytosolic and nuclear protein targets of thiol-reactive electrophiles. Chem Res Toxicol 2006; 19: 20-9.

29 Chen Q, Ngui JS, Doss GA, Wang RW, Cai X, DiNinno FP, et al. Cytochrome P450 3A4-mediated bioactivation of raloxifene: irreversible enzyme inhibition and thiol adduct formation. Chem Res Toxicol 2002; 15: 907-14.

30 Baer BR, Wienkers LC, Rock DA. Time-dependent inactivation of P450 3A4 by raloxifene: identification of Cys239 as the site of apoprotein alkylation. Chem Res Toxicol 2007; 20: 954-64.

31 Pearson JT, Wahlstrom JL, Dickmann LJ, Kumar S, Halpert JR, Wienkers LC, et al. Differential time-dependent inactivation of P450 $3 \mathrm{~A} 4$ and P450 3A5 by raloxifene: a key role for $\mathrm{C} 239$ in quenching reactive intermediates. Chem Res Toxicol 2007; 20: 1778-86.

32 Yukinaga H, Takami T, Shioyama SH, Tozuka Z, Masumoto H, Okazaki $\mathrm{O}$, et al. Identification of cytochrome P450 3A4 modification site with reactive metabolite using linear ion trap-Fourier transform mass spectrometry. Chem Res Toxicol 2007; 20: 1373-8.

33 Yu L, Liu H, Li W, Zhang F, Luckie C, van Breemen RB, et al. Oxidation of raloxifene to quinoids: potential toxic pathways via a diquinone methide and o-quinones. Chem Res Toxicol 2004; 17: 879-88.

34 Thompson DC, Perera K, Krol ES, Bolton JL. o-Methoxy-4-alkylphenols that form quinone methides of intermediate reactivity are the most toxic in rat liver slices. Chem Res Toxicol 1995; 8: 323-7. 